\title{
MÉTODO DE LAS RELACIONES CAUSALES PARA LA PLANIFICACIÓN DEL DESARROLLO SOSTENIBLE Aplicación en Pulpochoque
}

\author{
David Solano \\ Universidad ESAN \\ dsolano@esan.edu.pe \\ dadadi96@gmail.com
}

\begin{abstract}
Resumen
Los problemas que impiden el desarrollo sostenible de una sociedad son siempre producto de la interacción de muchos problemas-causa, los cuales pueden ser de naturaleza económica, sociocultural, tecnológica o política. Para plantear propuestas de solución adecuadas es necesario diferenciar entre problemas-causa y problemas-resultado; solo así se podrá lograr la sostenibilidad del trabajo por realizar. En este contexto, el Método de las Relaciones Causales brinda una herramienta para distinguir entre causas y consecuencias con base, principalmente, en las relaciones que se establecen entre los problemas. Este método puede ser utilizado para generar decisiones participativas que promuevan la acción integrada de una comunidad alrededor de los problemas que limitan su desarrollo.
\end{abstract}

Palabras clave: desarrollo, sostenibilidad, participación.

\begin{abstract}
The barrires to a society's sustainable development are typically the result of many problem-cause interactions, which may be of an economic, socio-cultural, technological or political nature. In order to find proper solutions, it is important to differentiate between problem-cause and problemresult relations; only with this method will it be possible to ensure sustainability. The Cause Relation Method is a tool that identifies causes and consequences starting with the relations between problems. This method may be used to generate participatory decisions that foster the integrated action of a community to address the constraints to its development.
\end{abstract}

Key words: development, sustainability, participation. 


\section{El método}

El Método de las Relaciones Causales para el Desarrollo Sostenible (RCDS) permite a los tomadores de decisiones identificar y graficar de manera participativa las relaciones de los problemas que impiden el desarrollo sostenible de un espacio determinado. Esto significa que puede ser aplicado a cualquier escala: una ciudad, un país, una empresa, etcétera.

El método ha sido inspirado en aquel empleado para generar los llamados síndromes de sostenibilidad del desarrollo (SSD), aunque tiene una finalidad y una utilidad diferentes. Los SSD son patrones funcionales de las relaciones sociedadambiente (Rabinovich y Torres, 2002). Una RCDS se convierte en un síndrome si es un patrón repetido en diferentes realidades. Bajo este concepto se ha identificado doce tipos de SSD, que representan diferentes relaciones causales repetidas (Rabinovich y Torres, 2002).

\section{Utilidad del método}

- Identificar participativamente, graficar y transmitir las relaciones causales entre problemas y la evolución de estos.

- Conocer cuáles son los problemas que se debe monitorear para saber si el resultado final será un incremento o una disminución del problema identificado.

- Desarrollar indicadores relevantes en función de los problemas con mayor capacidad de arrastre (CA) ya que, por definición, estos aquellos que generan más problemas.

\section{Limitaciones del método}

- Al solo graficar relaciones directas, no permite identificar problemas de conciencia, ya que estos no tienen relación directa con ningún problema específico. Sin embargo, es recomendable tenerlos en cuenta pues serán importantes para el resultado final, aunque su relación sea indirecta.

- Es muy trabajoso, pues requiere llenar gran cantidad de casilleros.

- Las relaciones identificadas debe ser demostradas empíricamente con información histórica.

\section{La secuencia de aplicación del método}

1. ${ }^{\circ}$ Identificar los problemas del desarrollo sostenible mediante la técnica de la lluvia de ideas. Dado que el objetivo es que el proceso sea participativo, se recomienda colocar todos los problemas que sean identificados. Estos pueden ser económicos, sociales, institucionales, tecnológicos $\mathrm{y} / \mathrm{o}$ ambientales.

2. ${ }^{\circ}$ Identificar las relaciones causales entre problemas a través de una «Matriz de relaciones causales». Para llenar esta matriz seguir este orden:

- Colocar en forma horizontal y vertical los problemas identificados.

- Cancelar la diagonal principal, ya que ningún problema se cotejará consigo mismo.

- Tomar el primer problema identificado y cotejarlo con el segundo problema. Se debe preguntar: «¿El problema 1 tiene influencia directa 
sobre el problema 2?», y, en función de la respuesta, hacer lo siguiente:

Si la respuesta es sí, colocar el número 1 en el casillero donde se cruzan los problemas 1 y 2 . Si la relación es negativa (cuando aumenta 1 disminuye 2), marcar este casillero, puede ser coloreándolo o rodeándolo con un círculo. Es importante colocar como 1 (positivo o negativo) solo las relaciones directas, es decir, cuando un problema influencia sobre otro por sí mismo, no a través de otros.

Si la respuesta es no, sea porque no hay relación o porque 2 origina 1 , colocar el número 0 .

3. ${ }^{\circ}$ Repetir la operación para todos los casilleros de la matriz, la cual tendrá un número de casilleros por llenar igual a (número de problemas) $^{2}-$ (número de $^{2}$ problemas).

4. ${ }^{\circ}$ Una vez llenada la matriz, se debe realizar la suma horizontal y la suma vertical. La suma horizontal representa la capacidad de un problema de originar otros (capacidad de arrastre, CA). La suma vertical significa la capacidad de un problema de ser originado por otros (capacidad de ser arrastrado, CSA).

5. Una vez realizadas las sumas, separar aquel o aquellos problemas que tengan un CA igual a 0 . Uno de ellos será el problema resultado final (PRF) que es producido por la interacción de todos los problemas identificados. Como se menciona en el punto 2..$^{\circ}$, colocar el número 0 significa que el problema es generado por otro o que no existe relación. Para poder identificar el resultado final de la interacción se selecciona aquel problema que tenga una CA igual a 0 combinado con la CSA más alta.
6. ${ }^{\circ}$ El problema identificado se convierte en el resultado final, tal como se representa en el primer nivel del diagrama.

7. ${ }^{\circ}$ Ahora se debe identificar el segundo nivel. Para establecer las relaciones directas se debe tomar aquellos problemas con una CA igual a 1 o 2 y que tengan una relación directa con el PRF. Para hacerlo, se observa la fila de los problemas mencionados con una CSA de 1 o 2, y se ve si alguno de estos tiene influencia sobre el PRF, es decir, si en el casillero donde se cruza este problema con el PRF aparece 1. El tener un solo problema o dos en este nivel facilitará las posteriores acciones de monitoreo. Si no existe un problema con estas características, se debe poner en el segundo nivel todos aquellos problemas que influyen directamente sobre el PRF. Se observa la columna del problema escogido y se destacan todos aquellos que se apareen con el número 1.

8. ${ }^{\circ}$ Para graficar el tercer nivel se debe identificar, con una metodología similar a la descrita en el punto 7, aquellos problemas que influyen en los problemas de segundo nivel.

9. ${ }^{\circ}$ Se recomienda no graficar más allá del cuarto o quinto nivel, aun cuando los problemas sean muchos más. Esto ayuda a corregir en parte la posibilidad de que las relaciones que se hayan identificado en la matriz no sean precisamente directas.

$10 .^{\circ}$ Se deben identificar indicadores para los problemas señalados, los cuales debe ser monitoreados. Para ello se recomienda elaborar una segunda matriz en la cual solo aparezcan los problemas que han sido identificados hasta el nivel del gráfico al que se haya llegado 
(cuarto o quinto nivel) y, en esta matriz, aquellos que tengan mayor CA son por definición los problemas prioritarios, aquellos que se debe solucionar en forma inmediata y monitorear. Dado que la matriz de relaciones indica la relación positiva (si aumenta uno, aumenta el otro) o negativa entre problemas, se podrá saber qué sucederá con el problema consecuencia si se incrementa o se reduce el problema causa.

\section{Aplicación del método en Pulpochoque}

A continuación se presenta información recabada en un caso real en forma participativa. Este ejemplo solo tiene como finalidad ilustrar cómo se puede usar la metodología y no presentar el caso con amplitud.

\subsection{Los problemas de Pulpochoque}

Pulpochoque se encuentra aproximadamente a 300 kilómetros al sur de la capital. Es una zona costera en la cual se extraen especies de valor comercial como erizo rojo (Loxechinus albus), chanque (Concholepas concholepas), lapa (Fissurella crassa), pulpo (Octopus mimus), choro (Aulacomya ater), aracanto (Lessonia sp.), caracol (Thais chocolata) y navaja (Tagelus dombeii), entre otros. En los últimos cinco años han disminuido las poblaciones de estas especies debido a la excesiva extracción.

En Pulpochoque, cuya actividad principal y de supervivencia es la pesca artesanal, se decretó, en 1991-1992, una veda para conservar especies en un ámbito de 20 kilómetros de extensión y, después de nueve meses, se obtuvo como resultado la repoblación de erizos y el aumento de su tamaño. Entonces, llegaron a Pulpochoque aproximadamente 50 embarcaciones marisqueras foráneas para extraer esta especie, iniciándose una depredación en gran escala de la biodiversidad local.

Entre los años 1990 y 2000 se formaron 15 asociaciones de pescadores embarcados y no embarcados (pulmoneros), las cuales siguen funcionando hasta ahora. Estas iniciaron un manejo conjunto del ámbito de 20 kilómetros en torno a Pulpochoque.

Cada asociación asumió la responsabilidad de cuidar una zona territorial para repoblar erizos, chanques y pulpos y lograr un uso sostenible de estas especies. Se realizaron trabajos comunales y se construyeron pozas seminaturales aprovechando la baja marea. También se llevaron adelante experiencias de engorde en sistemas suspendidos.

Al no contar con información sobre la crianza de estas especies, se logró su reproducción en forma empírica en pozas. Esta experiencia tiene problemas por la interferencia de otras personas sobre las zonas de trabajo, a través de robos (a pesar de montar guardia las 24 horas del día), ya que no cuentan con la posesión legal de las áreas y los recursos.

En un trabajo participativo con los pescadores y las autoridades de la zona se han detectado los siguientes problemas que afectan el desarrollo sostenible:

P1. Ineficiencia en los procesos de carnetización de los pescadores. Los pescadores no pueden acceder a un carnet por el costo de los trámites. Además, los trámites son largos, lo cual origina que se entreguen los carnets en fecha muy próxima a su expiración, con la consiguiente necesidad de repetir trámites y gastos. 
P2. Marco normativo poco claro. No existe claridad en los pescadores en cuanto a los trámites necesarios para las acciones de repoblamiento.

P3. Debilidad de las acciones de control $y$ vigilancia. Escasas acciones de control sobre el respeto a las normas en mar y en costa por parte de Capitanía del Puerto, la Municipalidad de Pulpochoque y el Viceministerio de Pesquería.

P4. Percepción de que las normas no se basan en la realidad actual. Las normas no son aplicables a la realidad local, ya que no recogen las necesidades y las posibilidades de los pescadores.

P5. Procesos de difusión deficientes por parte del Estado. Los encargados de difundir las normas, pertenecientes al Viceministerio de Pesquería, han generado confusión entre los pescadores y han exigido requisitos inaplicables, por desconocimiento e interpretación errónea de las normas existentes. Además, esta difusión no ha tenido la frecuencia requerida.

P6. Organizaciones no formalizadas. Existen 15 organizaciones, de las cuales ocho están formalizadas y siete, en proceso de formalización. En la misma situación se encuentra la Comunidad Pesquera de Pulpochoque (COPP).

P7. Escasa inversión en proyectos de repoblamiento. Escasez de apoyo técnico y financiero para llevar a cabo las acciones de repoblamiento emprendidas por los pescadores artesanales.

P8. Escasez de capacitación y de alternativas para la participación familiar en los recursos productivos. No existen registros de acciones de capacitación a las familias de los pescadores sobre actividades alternativas, como la artesanía.

P9. Inexistente asignación de derechos de recursos y áreas. El no existir propiedad sobre los recursos que se han cultivado en pozas ha originado que no se puedan defender ni recibir el apoyo efectivo de las autoridades competentes para evitar robos y la intervención de terceros sobre el área de trabajo de cada asociación de pescadores.

P10. Interferencia entre artes de pesca. Existen cruces en áreas comunes entre bolichitos foráneos (con equipo sofisticado) y pescadores artesanales, lo que origina conflictos entre actividades que compiten entre sí por el recurso.

P11. Escasez e inaccesibilidad de información. Los pescadores artesanales no tienen fácil acceso a las investigaciones generadas.

P12. Escasez de especies por causas naturales (Fenómeno El Niño). El calentamiento de las aguas genera escasez de plancton, lo cual origina la migración de las especies comerciales.

P13. Contaminación del mar por aguas residuales domésticas. Los desagües domésticos del campamento minero cercano son arrojados al mar sin previo tratamiento, lo cual contamina el mar.

P14. Excesiva presión sobre las especies hidrobiológicas marino-costeras. Por necesidad, se extraen especies juveniles, lo que impide que completen su ciclo de vida hasta alcanzar un valor comercial apropiado y su reproducción exitosa. 
P15. Contaminación minera. Existe el riesgo de que las descargas por la actividad minera (que se desarrolla en una zona cercana) y los pasivos ambientales puedan afectar la zona adyacente al ámbito del programa, lo cual podría generar alteraciones en las condiciones sanitarias de los recursos sujetos a manejo y cultivo e influir en la percepción del mercado potencial.

P16. Alteración de los ecosistemas por tecnologías inadecuadas de pesca. Por ejemplo, el uso de dinamita para la pesca que ahuyenta las especies y las elimina en forma masiva.

P17. Desconocimiento de la experiencia empírica de los pescadores artesanales. El Estado y la cooperación internacional no han apoyado ni aprovechado para su difusión las experiencias exitosas de los pescadores, en parte por falta de conocimiento acerca de ellas y en parte por falta de fondos o por no considerarlas relevantes.

P18. Contaminación debida a la pesca artesanal (embarcados y no embarcados). La pesca artesanal genera procesos de contaminación por combustibles y aceites que se arrojan al mar, al igual que desperdicios sólidos y líquidos que se echan a la playa y el mar.

P19. Desconocimiento de los mercados internacionales. No existe conocimiento de los precios, los mercados y las posibilidades de las especies hidrobiológicas priorizadas en el Programa Piloto.

P20. Inexistente delimitación georreferenciada de los bancos naturales. Existe solo un conocimiento empírico de la ubicación de los bancos naturales.

P21. Escaso desarrollo de alternativas económicas. No se ha profundizado el trabajo sobre actividades alternativas a la pesca artesanal como el ecoturismo y la artesanía.

P22. Escasa participación de los pescadores en la planificación. Los puntos de vista de los pescadores no son considerados para planificar el uso de las zonas marino-costeras.

P23. Migraciones de la sierra a la costa. En la década de 1980, y por causa del terrorismo, gran cantidad de migrantes de diversas partes de la sierra sur migraron a Pulpochoque en busca de trabajo y se dedicaron a la actividad pesquera.

P24. Inadecuadas políticas de promoción de la actividad pesquera acuícola. Por parte de la autoridad pesquera del país.

P25. Inversiones mineras. A 5 kilómetros de Pulpochoque se desarrolla una actividad minera de gran envergadura.

Dada la amplitud de los problemas y la diversidad de estos, se busca determinar qué problemas deberían ser priorizados para abordarlos y buscarles solución.

\subsection{La solución del caso}

1. ${ }^{\circ}$ Desarrollar la Matriz de Relaciones Causales. Los números en cursivas representan relaciones inversas o negativas. Las letras I, relaciones indirectas importantes (ver gráfico 1). 


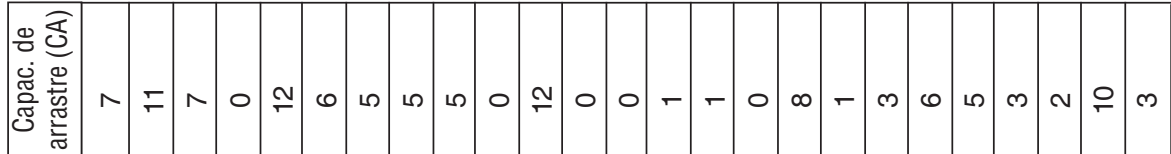

N

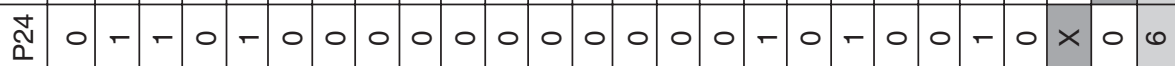
ֻ

ก

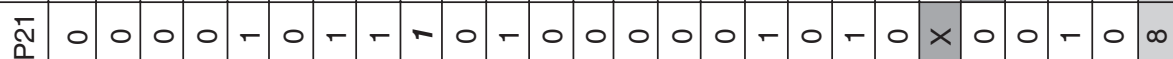
ลิ

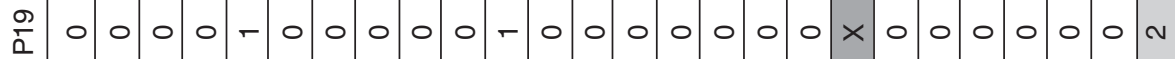

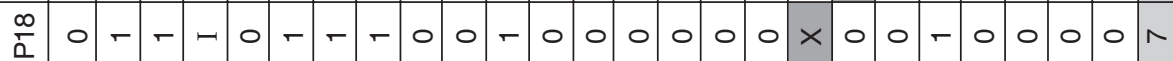

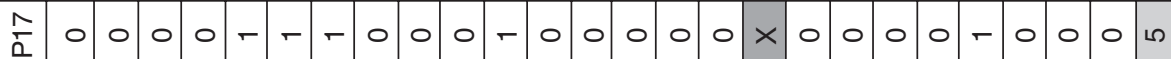

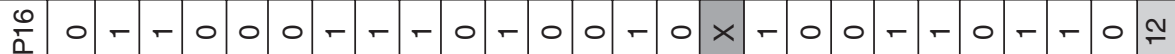

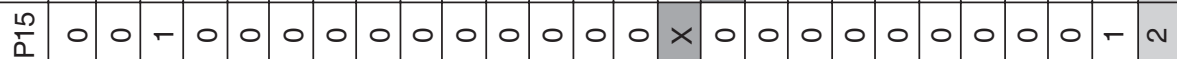

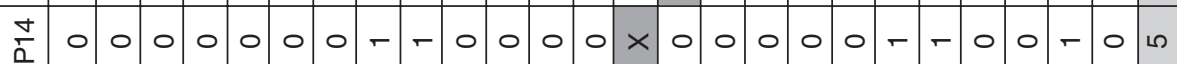

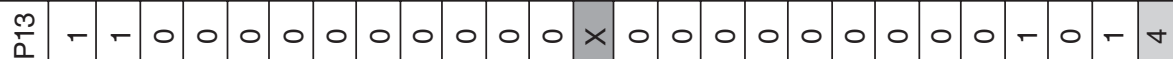

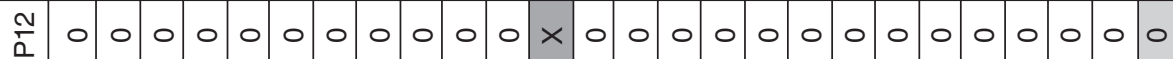

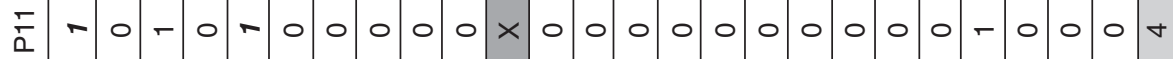

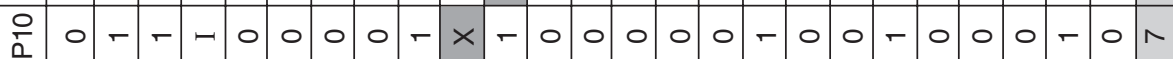

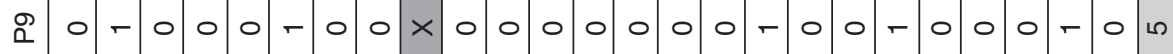
$\infty \begin{array}{lllllllllllllllllllllllllll}0 & \infty & 0 & 0 & 0 & - & - & - & \times & 0 & 0 & 0 & 0 & 0 & 0 & 0 & 0 & - & 0 & 0 & 0 & - & 0 & 0 & - & 0 & \Lambda\end{array}$

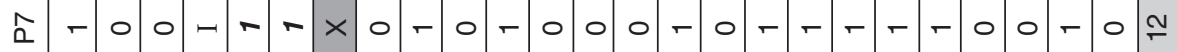

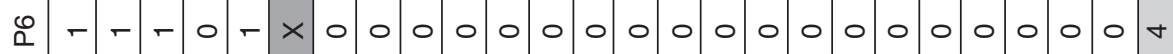
ю

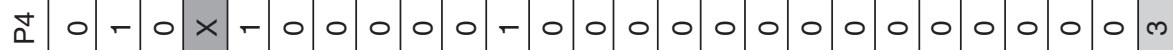
m

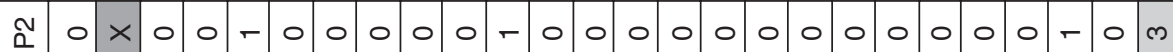

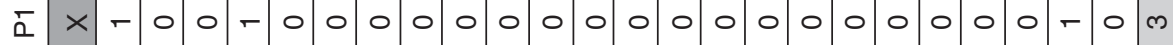
¿் 
2. ${ }^{\circ}$ Identificar aquel problema con CA igual a 0 y la mayor CSA. Este problema es P16 «Alteración de ecosistemas por tecnologías inadecuadas de pesca», que tiene una CA igual a 0 y una CSA igual a 12, una de los mayores en este rubro. P16 se convierte en el «problema resultado final» (PRF). El otro problema con $\mathrm{CA}$ igual a 0 (P10 «Interferencia entre artes de pesca»), por citar un ejemplo, tiene una CSA igual a 7. Así tenemos el primer nivel.

3. ${ }^{\circ}$ Identificar el problema con relación más directa con el PRF. Este es P14 «Excesiva presión sobre las especies hidrobiológicas marino-costeras», que tiene una $\mathrm{CA}$ de 1 y cuya única relación de arrastre se da con P16 (ver gráfico 2).

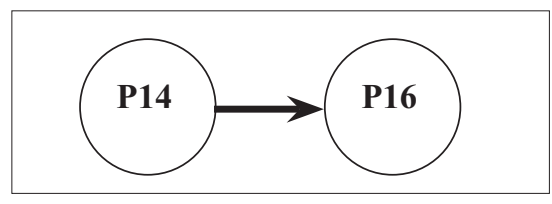

Gráfico 2

Problema con relación más directa con el PRF

4. ${ }^{\circ}$ Identificar el tercer nivel. Se busca en la columna de P14 cuáles son los problemas que influyen sobre este directamente y se encuentra los siguientes (ver gráfico 3):

P8. Escasa capacitación

P9. Inexistente asignación de derechos de recursos y áreas

P21. Escaso desarrollo de alternativas económicas

P24. Inadecuadas políticas de promoción de la actividad pesquera acuícola

5..$^{\circ}$ Identificar el cuarto nivel. Se observa en la columna de cada uno de los pro-

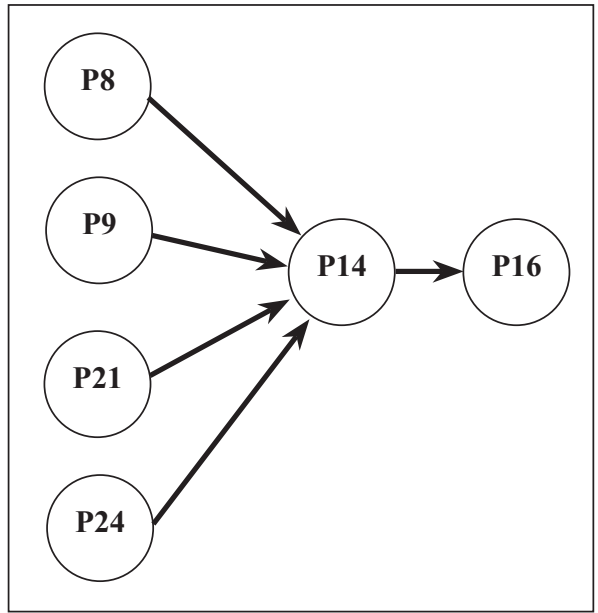

Gráfico 3

Identificación del tercer nivel

blemas del tercer nivel y se identifica cuáles son aquellos que los influencian directamente. Así se tiene la siguiente lista (ver gráfico 4):

\section{A P8. ESCASA CAPACITACIÓN}

P1. Carnetización

P5. Escasa difusión

P6. Organizaciones no formalizadas

P7. Escasas inversiones en repoblamiento

P17. Desconocimiento de experiencia empírica

P21. Escaso desarrollo de alternativas

$\mathrm{P} 24$. Inadecuadas políticas

\section{A P9. ESCASA ASIGNACIÓN DE RECURSOS Y ÁREAS}

P2. Marco normativo

P6. Organizaciones no formalizadas

P17. Desconocimiento de experiencia empírica

P20. Inexistente delimitación georreferenciada

P24. Inadecuadas políticas 


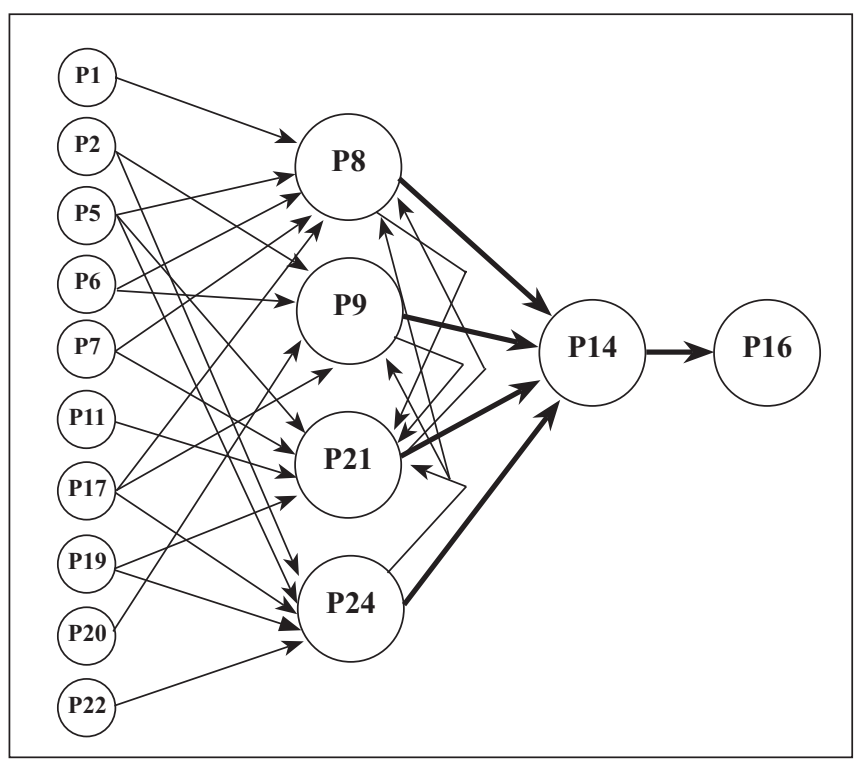

Gráfico 4

Identificación del cuarto nivel

\section{A P21. ESCASO DESARROLLO DE ALTERNATIVAS}

P5. Escasa difusión

P7. Escasas inversiones en repoblamiento

P8. Escasa capacitación

P9. Escasa asignación de recursos y áreas

P11. Escasa información

P17. Desconocimiento de experiencia empírica

P19. Desconocimiento de mercados internacionales

$\mathrm{P} 24$. Inadecuadas políticas

A P24. INADECUADAS POLÍTICAS

P2. Marco normativo

P3. Debilidad de control

P5. Escasa difusión

P17. Desconocimiento de experiencia empírica
P19. Desconocimiento de mercados internacionales

P22. Escasa participación de pescadores en planificación

Como se puede ver en esta lista, las relaciones causales son cada vez más cruzadas. Sin embargo, ofrece una idea de la interrelación entre los diferentes problemas. Por ejemplo, destaca el hecho de que los problemas de tercer nivel se entrecruzan. La decisión más práctica es permanecer en este nivel, considerando que están representados 17 de los 24 problemas esbozados. Estos 17 serían los problemas priorizados en función de su influencia directa sobre los demás.

$6^{\circ} \mathrm{Al}$ aplicar un método gráfico se aprecia que existen problemas que actúan como los desencadenantes más importantes del proceso que da como resultado 
final «Alteración de ecosistemas por tecnologías inadecuadas de pesca». Estos problemas serían P5 «Difusión deficiente», P17 «Desconocimiento de experiencia empírica» y P24 «Inadecuadas políticas», ya que son los problemas que establecen más relaciones de causalidad con los otros. Esto no quiere decir que los otros no son importantes sino que influyen en menor medida en el flujo de causalidad planteado. Esto se puede comprobar en una matriz donde solo aparezcan los problemas priorizados, como la que se presenta a continuación (ver gráfico 5).

Como puede observarse, los problemas con alta influencia sobre los demás son P5 $(\mathrm{CA}=11), \mathrm{P} 17(\mathrm{CA}=7)$ y $\mathrm{P} 24(\mathrm{CA}=8)$. Aparece también P11 «Escasez e inaccesibilidad de información», como un problema importante a solucionar y monitorear. Estos serían los problemas priorizados por su alta capacidad de influir en otros, tal como está definida la capacidad de arrastre.

Como resultado de este proceso se pueden tomar las siguientes importantes decisiones:

1. ${ }^{\circ}$ El resultado de la interacción de todos los problemas es «Alteración de ecosistemas por tecnologías inadecuadas de pesca». Es el tema que se debe buscar resolver, para ello se tiene que desarrollar indicadores que permitan conocer la evolución del problema.

2. ${ }^{\circ}$ Los temas prioritarios son los signados como P5, P11, P17 y P24. Estos tienen gran capacidad de influir sobre los otros por lo que es prioritario tomar decisiones sobre ellos y plantear planes de trabajo para buscar superarlos. Son además aquellos donde deben realizarse mayores inversiones y esfuerzos.

3. ${ }^{\circ}$ Toda decisión que se tome sobre estos problemas va a tener consecuencias sobre otros problemas. Se debe tener en cuenta la naturaleza de estas relaciones para evitar resultados diferentes a los esperados.

\section{Conclusión}

El Método de las Relaciones Causales es una metodología que puede usarse para la planificación participativa del desarrollo sostenible, pues permite priorizar enfoques. También es una buena forma de transmitir resultados y relaciones a la población, con el propósito de involucrarla en la toma de decisiones y en las acciones posteriores para lograr el desarrollo. 


\begin{tabular}{|c|c|c|c|c|c|c|c|c|c|c|c|c|c|c|c|c|c|}
\hline 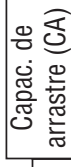 & 10 & 0 & $\mp$ & 10 & $\nabla$ & $\nabla$ & $\nabla$ & $\infty$ & - & 0 & $\wedge$ & m & $\nabla$ & $\nabla$ & ल & $\infty$ & \\
\hline ্ָ & 0 & - & - & 0 & 0 & 0 & 0 & 0 & 0 & 0 & - & - & 0 & 0 & - & $\times$ & 10 \\
\hline $\begin{array}{l}\text { న } \\
\Sigma\end{array}$ & - & 0 & - & - & 0 & - & 0 & - & 0 & 0 & 0 & 0 & 0 & 0 & $\times$ & 0 & 10 \\
\hline $\bar{\Sigma}$ & 0 & 0 & - & 0 & - & - & - & - & 0 & 0 & - & - & 0 & $\times$ & 0 & - & $\infty$ \\
\hline $\begin{array}{l}\stackrel{0}{ } \\
\text { ¿ }\end{array}$ & 0 & 0 & 0 & 0 & 0 & 0 & 0 & 0 & 0 & 0 & 0 & 0 & $\times$ & 0 & 0 & 0 & 0 \\
\hline$\frac{\sigma}{\square}$ & 0 & 0 & - & 0 & 0 & 0 & 0 & - & 0 & 0 & 0 & $\times$ & 0 & 0 & 0 & 0 & $\sim$ \\
\hline$\frac{1}{\alpha}$ & 0 & 0 & - & - & - & 0 & 0 & - & 0 & 0 & $\times$ & 0 & 0 & 0 & - & 0 & 10 \\
\hline$\frac{0}{2}$ & 0 & - & 0 & 0 & - & - & - & - & - & $\times$ & - & 0 & - & - & 0 & - & 으 \\
\hline$\frac{\nabla}{\square}$ & 0 & 0 & 0 & 0 & 0 & - & - & 0 & $\times$ & 0 & 0 & 0 & - & - & 0 & - & 10 \\
\hline $\bar{\square}$ & - & 0 & - & 0 & 0 & 0 & 0 & $\times$ & 0 & 0 & 0 & 0 & 0 & 0 & - & 0 & m \\
\hline ه & 0 & - & 0 & - & 0 & 0 & $\times$ & 0 & 0 & 0 & - & 0 & - & 0 & 0 & - & 10 \\
\hline$\stackrel{\infty}{\alpha}$ & - & 0 & - & - & - & $\times$ & 0 & 0 & 0 & 0 & - & 0 & 0 & - & 0 & - & $\wedge$ \\
\hline$\hat{\alpha}$ & - & 0 & - & - & $\times$ & 0 & - & - & 0 & 0 & - & - & - & - & 0 & - & 음 \\
\hline 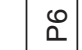 & - & - & - & $\times$ & 0 & 0 & 0 & 0 & 0 & 0 & 0 & 0 & 0 & 0 & 0 & 0 & $m$ \\
\hline$\stackrel{\circ}{\circ}$ & 0 & - & $\times$ & 0 & 0 & 0 & 0 & - & 0 & 0 & - & 0 & 0 & 0 & 0 & 0 & $m$ \\
\hline$\approx$ & 0 & $\times$ & - & 0 & 0 & 0 & 0 & - & 0 & 0 & 0 & 0 & 0 & 0 & 0 & - & $m$ \\
\hline $\bar{a}$ & $\times$ & - & - & 0 & 0 & 0 & 0 & 0 & 0 & 0 & $\circ$ & 0 & 0 & 0 & 0 & - & $m$ \\
\hline & $\bar{\alpha}$ & ¿ & $\stackrel{10}{\alpha}$ & $\stackrel{0}{0}$ & $\hat{a}$ & $\begin{array}{l}\infty \\
0\end{array}$ & ๑ & $\bar{\alpha}$ & $\frac{\nabla}{a}$ & $\frac{0}{a}$ & $\frac{\hat{\sigma}}{a}$ & $\frac{\sigma}{2}$ & 尺ે & $\bar{\aleph}$ & స్ & $\stackrel{\sim}{\sim}$ & 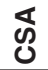 \\
\hline
\end{tabular}




\section{Referencias bibliográficas}

GALLOPIN, Gilberto. 2004. Los síndromes de sostenibilidad del desarrollo. Buenos Aires: Cepal. Material de Curso Indicadores de Desarrollo Sostenible en América Latina.

2006. Los indicadores de desarrollo sostenible: aspectos conceptuales y metodológicos. Santiago de Chile: Cepal. Ponencia para el Seminario de Expertos sobre Indicadores de Sostenibilidad.
RABIONOVICH, Jorge y TORRES, Filemón. 2003. Caracterización de los síndromes de sostenibilidad del desarrollo. Santiago de Chile: Comisión Económica para América Latina.

SOLANO, David. 2003. Cómo planificar la acción ambiental. Lima: ESAN. Material del curso Gestión Ambiental para el Desarrollo Sostenible Local (no publicado). 